\title{
VARIANCE ESTIMATES FOR RANDOM DISC-POLYGONS IN SMOOTH CONVEX DISCS
}

\author{
FERENC FODOR *** AND \\ VIKTOR VíGH, ${ }^{* * * *}$ University of Szeged
}

\begin{abstract}
In this paper we prove asymptotic upper bounds on the variance of the number of vertices and the missed area of inscribed random disc-polygons in smooth convex discs whose boundary is $C_{+}^{2}$. We also consider a circumscribed variant of this probability model in which the convex disc is approximated by the intersection of random circles.
\end{abstract}

Keywords: Disc-polygon; random approximation; variance

2010 Mathematics Subject Classification: Primary 52A22

Secondary 60D05

\section{Introduction and results}

Let $K$ be a convex disc (compact convex set with nonempty interior) in the Euclidean plane $\mathbb{R}^{2}$. We use the notation $B^{2}$ for the origin-centred unit-radius closed circular disc, and $S^{1}$ for its boundary, the unit circle. The area of Lebesgue measurable subsets of $\mathbb{R}^{2}$ is denoted by $A(\cdot)$. Assume that the boundary $\partial K$ is of class $C_{+}^{2}$, that is, two times continuously differentiable and the curvature at every point of $\partial K$ is strictly positive. Let $\kappa(x)$ denote the curvature at $x \in \partial K$, and let $\kappa_{\mathrm{m}}\left(\kappa_{\mathrm{M}}\right)$ be the minimum (maximum) of $\kappa(x)$ over $\partial K$. It is known (see [29, Section 3.2]) that in this case a closed circular disc of radius $r_{\mathrm{m}}=1 / \kappa_{\mathrm{M}}$ rolls freely in $K$, that is, for each $x \in \partial K$, there exists a $p \in \mathbb{R}^{2}$ with $x \in r_{\mathrm{m}} B^{2}+p \subset K$. Moreover, $K$ slides freely in a circle of radius $r_{\mathrm{M}}=1 / \kappa_{\mathrm{m}}$, which means that for each $x \in \partial K$ there is a vector $p \in \mathbb{R}^{2}$ such that $x \in r_{\mathrm{M}} \partial B^{2}+p$ and $K \subset r_{\mathrm{M}} B^{2}+p$. The latter yields that for any two points $x, y \in K$, the intersection of all closed circular discs of radius $r \geq r_{\mathrm{M}}$ containing $x$ and $y$, denoted by $[x, y]_{r}$ and called the $r$-spindle of $x$ and $y$, is also contained in $K$. Furthermore, for any $X \subset K$, the intersection of all radius $r \geq r_{\mathrm{M}}$ circles containing $X$, called the closed $r$-hyperconvex hull (or $r$-hull for short) and denoted by $\operatorname{conv}_{r}(X)$, is contained in $K$. The concept of hyperconvexity, also called spindle convexity or $r$-convexity, can be traced back to Mayer [21]. For a systematic treatment of geometric properties of hyperconvex sets and further references, see, for example, [10] and [19], and in a more general setting [20]. The notion of convexity arises naturally in many questions where a convex set can be represented as the intersection of equal radius closed balls. As recent examples of such problems, we mention the Kneser-Poulsen conjecture; see, for example, [7]-[9], and inequalities for intrinsic volumes in [22]. A more complete list can be found in [10], for short overviews, see also [15], [16], and [18].

Let $K$ be a convex disc with $C_{+}^{2}$ boundary, and let $x_{1}, x_{2}, \ldots$ be independent random points chosen from $K$ according to the uniform probability distribution, and write $X_{n}=\left\{x_{1}, \ldots, x_{n}\right\}$.

Received 7 February 2018; revision received 17 August 2018.

* Postal address: Bolyai Institute, University of Szeged, Aradi vértanúk tere 1, 6720 Szeged, Hungary.

** Email address: fodorf@math.u-szeged.hu

*** Email address: vigvik@ math.u-szeged.hu 
The classical convex hull conv $\left(X_{n}\right)$ is a random convex polygon in $K$. The geometric properties of $\operatorname{conv}\left(X_{n}\right)$ have been investigated extensively in the literature. For more information on this topic and further references we refer the reader to the surveys [1], [28], [30], [37], and the book [31].

Here we examine the following random model. Let $r \geq r_{\mathrm{M}}$, and let $K_{n}^{r}=\operatorname{conv}_{r}\left(X_{n}\right)$ be the $r$-hull of $X_{n}$, which is a (uniform) random disc-polygon in $K$. Let $f_{0}\left(K_{n}^{r}\right)$ denote the number of vertices (and also the number of edges) of $K_{n}^{r}$, and let $A\left(K_{n}^{r}\right)$ denote the area of $K_{n}^{r}$. The asymptotic behaviour of the expectation of the random variables $A\left(K_{n}^{r}\right)$ and $f_{0}\left(K_{n}^{r}\right)$ was investigated by Fodor et al. [18], where (among others) the following two theorems were proved.

Theorem 1. (Fodor et al. [18, Theorem 1.1, p. 901].) Let $K$ be a convex disc whose boundary is of class $C_{+}^{2}$. For any $r>r_{\mathrm{M}}$, it holds that

$$
\lim _{n \rightarrow \infty} \mathbb{E}\left(f_{0}\left(K_{n}^{r}\right)\right) n^{-1 / 3}=\sqrt[3]{\frac{2}{3 A(K)}} \Gamma\left(\frac{5}{3}\right) \int_{\partial K}\left(\kappa(x)-\frac{1}{r}\right)^{1 / 3} \mathrm{~d} x,
$$

and

$$
\lim _{n \rightarrow \infty} \mathbb{E}\left(A\left(K \backslash K_{n}^{r}\right)\right) n^{2 / 3}=\sqrt[3]{\frac{2 A(K)^{2}}{3}} \Gamma\left(\frac{5}{3}\right) \int_{\partial K}\left(\kappa(x)-\frac{1}{r}\right)^{1 / 3} \mathrm{~d} x .
$$

Theorem 2. (Fodor et al. [18, Theorem 1.2, Equation (1.7), p. 901].) For $r>0$, let $K=r B^{2}$ be the closed circular disc of radius $r$. Then

$$
\lim _{n \rightarrow \infty} \mathbb{E}\left(f_{0}\left(K_{n}^{r}\right)\right)=\frac{1}{2} \pi^{2}
$$

and

$$
\lim _{n \rightarrow \infty} \mathbb{E}\left(A\left(K \backslash K_{n}^{r}\right)\right) n=\frac{1}{3} r^{2} \pi^{3} .
$$

We denote by $\Gamma(\cdot)$ Euler's gamma function, and integration on $\partial K$ is with respect to arclength.

Observe that in Theorem 2 the expectation $\mathbb{E}\left(f_{0}\left(K_{n}^{r}\right)\right)$ of the number of vertices tends to a constant as $n \rightarrow \infty$. This is a surprising fact that has no clear analogue in the classical convex case. A similar phenomenon was recently established in [6] concerning the expectation of the number of facets of certain spherical random polytopes in halfspheres; see [6, Theorem 3.1].

We note that Theorem 1 can also be considered as a generalization of the classical asymptotic results of Rényi and Sulanke about the expectation of the vertex number and missed area of classical random convex polygons in smooth convex discs (see [25], [26]) in the sense that it reproduces the formulas of Rényi and Sulanke in the limit as $r \rightarrow \infty$; see [18, Section 3].

Obtaining information on the second-order properties of random variables associated with random polytopes is much more difficult than on first-order properties. It is only recently that variance estimates, laws of large numbers, and central limit theorems have been proved in various models; see, for example, [2]-[5], [13], [17], [23], [24], and [32]-[36]. For an overview, see [1] and [30].

In this paper we prove the following asymptotic estimates for the variance of $f_{0}\left(K_{n}^{r}\right)$ and $A\left(K_{n}^{r}\right)$ in the spirit of Reitzner [23].

For the order of magnitude, we use the following common symbols: if for two functions $f, g: I \rightarrow \mathbb{R}, I \subset \mathbb{R}$, there is a constant $\gamma>0$ such that $|f| \leq \gamma g$ on $I$, then we write $f \ll g$ or $f=O(g)$. If $f \ll g$ and $g \ll f$, then this fact is indicated by the notation $f \approx g$. 
Theorem 3. With the same hypotheses as in Theorem 1, it holds that

$$
\operatorname{var}\left(f_{0}\left(K_{n}^{r}\right)\right) \ll n^{1 / 3},
$$

and

$$
\operatorname{var}\left(A\left(K_{n}^{r}\right)\right) \ll n^{-5 / 3},
$$

where the implied constants depend only on $K$ and $r$.

In the special case when $K$ is the closed circular disc of radius $r$, we prove the following theorem.

Theorem 4. With the same hypotheses as in Theorem 2, it holds that

$$
\operatorname{var}\left(f_{0}\left(K_{n}^{r}\right)\right) \approx \text { constant }
$$

and

$$
\left.\operatorname{var}\left(A\left(K_{n}^{r}\right)\right)\right) \ll n^{-2},
$$

where the implied constants depend only on $r$.

From Theorem 3 we can conclude the following strong laws of large numbers. Since the proof follows a standard argument based on Chebysev's inequality and the Borel-Cantelli lemma (see, for example, [13, p. 2294] or [23, Section 5], and [3, p. 174]), we omit the details.

Theorem 5. With the same hypotheses as in Theorem 1, it holds with probability 1 that

$$
\lim _{n \rightarrow \infty} f_{0}\left(K_{n}^{r}\right) n^{-1 / 3}=\sqrt[3]{\frac{2}{3 A(K)}} \Gamma\left(\frac{5}{3}\right) \int_{\partial K}\left(\kappa(x)-\frac{1}{r}\right)^{1 / 3} \mathrm{~d} x,
$$

and

$$
\lim _{n \rightarrow \infty} A\left(K \backslash K_{n}^{r}\right) n^{2 / 3}=\sqrt[3]{\frac{2 A(K)^{2}}{3}} \Gamma\left(\frac{5}{3}\right) \int_{\partial K}\left(\kappa(x)-\frac{1}{r}\right)^{1 / 3} \mathrm{~d} x .
$$

In the theory of random polytopes there is more information on models in which the polytopes are generated as the convex hull of random points from a convex body $K$ than on polyhedral sets produced by random closed half-spaces containing $K$. For some recent results and references in this direction, see, for example, [11], [12], [17], and the survey [30].

In Section 5 we consider a model of random disc-polygons that contain a given convex disc with $C_{+}^{2}$ boundary. In this circumscribed probability model, we give asymptotic formulas for the expectation of the number of vertices of the random disc-polygon, and the area difference and the perimeter difference of the random disc-polygon and $K$; see Theorem 6 . Furthermore, Corollary 1 provides an asymptotic upper bound on the variance of the number of vertices of the circumscribed random polygons.

The outline of the paper is as follows. In Section 2 we collect some geometric facts that are needed for the arguments. Theorem 3 is proved in Section 3, and Theorem 4 is verified in Section 4. In Section 5 we discuss a different probability model in which $K$ is approximated by the intersection of random closed circular discs containing $K$. This model is a kind of dual to the inscribed one. 


\section{Preparations}

We note that it is enough to prove Theorem 3 for the case when $r_{M}<1$ and $r=1$, and Theorem 4 for $r=1$. The general statements then follow by a simple scaling argument. Therefore, from now on we assume that $r=1$ and to simplify notation we write $K_{n}$ for $K_{n}^{1}$.

Let $\bar{B}^{2}$ denote the open unit ball of radius 1 centred at the origin $o$. A disc-cap (of radius 1) of $K$ is a set of the form $K \backslash\left(\bar{B}^{2}+p\right)$ for some $p \in \mathbb{R}^{2}$.

We start with recalling the following notation from [18]. Let $x$ and $y$ be two points from $K$. The two unit circles passing through $x$ and $y$ determine two disc-caps of $K$, which we denote by $D_{-}(x, y)$ and $D_{+}(x, y)$, respectively, such that $A\left(D_{-}(x, y)\right) \leq A\left(D_{+}(x, y)\right)$. For brevity of notation, we write $A_{-}(x, y)=A\left(D_{-}(x, y)\right)$ and $A_{+}(x, y)=A\left(D_{+}(x, y)\right)$. In $[18$, see Lemma 3] it was shown that if the boundary of $K$ is of class $C_{+}^{2}\left(r_{\mathrm{M}}<1\right)$ then there exists a $\delta>0$ (depending only on $K$ ) with the property that for any $x, y \in$ int $K$, it holds that $A_{+}(x, y)>\delta$.

We need some further technical lemmas about general disc-caps. Let $u_{x} \in S^{1}$ denote the (unique) outer unit normal to $K$ at the boundary point $x$, and $x_{u} \in \partial K$ the unique boundary point with outer unit normal $u \in S^{1}$.

Lemma 1. (Fodor et al. [18, Lemma 4.1, p. 905].) Let $K$ be a convex disc with $C_{+}^{2}$ smooth boundary and assume that $\kappa_{\mathrm{m}}>1$. Let $D=K \backslash\left(\bar{B}^{2}+p\right)$ be a nonempty disc-cap of $K$ (as above). Then there exists a unique point $x_{0} \in \partial K \cap \partial D$ such that there exists a $t \geq 0$ with $B^{2}+p=B^{2}+x_{0}-(1+t) u_{x_{0}}$. We refer to $x_{0}$ as the vertex of $D$ and to $t$ as the height of $D$.

Let $D(u, t)$ denote the disc-cap with vertex $x_{u} \in \partial K$ and height $t$. Note that for each $u \in S^{1}$, there exists a maximal positive constant $t^{*}(u)$ such that $\left(B+x_{u}-(1+t) u\right) \cap K \neq \varnothing$ for all $t \in\left[0, t^{*}(u)\right]$. For simplicity, we let $A(u, t)=A(D(u, t))$ and let $\ell(u, t)$ denote the arc-length of $\partial D(u, t) \cap\left(\partial B+x_{u}-(1+t) u\right)$.

We need the following limit relations about the behaviour of $A(u, t)$ and $\ell(u, t)$ which we recall from [18, Lemma 4.2, p. 905]:

$$
\lim _{t \rightarrow 0^{+}} \ell\left(u_{x}, t\right) t^{-1 / 2}=2 \sqrt{\frac{2}{\kappa(x)-1}}, \quad \lim _{t \rightarrow 0^{+}} A\left(u_{x}, t\right) t^{-3 / 2}=\frac{4}{3} \sqrt{\frac{2}{\kappa(x)-1}} .
$$
in $u$ :

It is clear that (6) implies that $A(u, t)$ and $\ell(u, t)$ satisfy the following relations uniformly

$$
\ell\left(u_{x}, t\right) \approx t^{1 / 2}, \quad A\left(u_{x}, t\right) \approx t^{3 / 2},
$$

where the implied constants depend only on $K$.

Let $D$ be a disc-cap of $K$ with vertex $x$. For a line $e \subset \mathbb{R}^{2}$ with $e \perp u_{x}$, let $e_{+}$denote the closed half-plane containing $x$. Then there exist a maximal cap $C_{-}(D)=K \cap e_{+} \subset D$, and a minimal cap $C_{+}(D)=e_{+}^{\prime} \cap K \supset D$.

Claim 1. There exists a constant $\hat{c}$ depending only $K$ such that if the height of the disc-cap $D$ is sufficiently small, then

$$
\hat{c}\left(C_{-}(D)-x\right) \supset\left(C_{+}(D)-x\right) .
$$

Proof. Denote by $h_{-}\left(h_{+}\right)$the height of $C_{-}(D)\left(C_{+}(D)\right.$, respectively), which is the distance of $x$ and $e$ ( $e^{\prime}$, respectively). By convexity, it is enough to find a constant $\hat{c}>0$ such that for all disc-caps of $K$ with sufficiently small height $h_{+} / h_{-}<\hat{c}$ holds.

Choose an arbitrary $R \in\left(1 / \kappa_{\mathrm{m}}, 1\right)$, and consider $\hat{B}=R B^{2}+x-R u_{x}$, the disc of radius $R$ that supports $K$ in $x$. Clearly, $\hat{B} \supset K$ implies that $D=K \cap\left(\bar{B}^{2}+p\right) \subset\left(\hat{B} \cap\left(\bar{B}^{2}+p\right)=\hat{D}\right.$. 
Also, for the respective heights $\hat{h}_{-}$and $\hat{h}_{+}$of $C_{-}(\hat{D})$ and $C_{+}(\hat{D})$, we have $\hat{h}_{-}=h_{-}$and $\hat{h}_{+}>h_{+}$. Thus, it is enough to find $\hat{c}$ such that $\hat{h}_{+} / \hat{h}_{-}<\hat{c}$. The existence of such $\hat{c}$ is clear from elementary geometry.

Let $x_{i}, x_{j}(i \neq j)$ be two points from $X_{n}$, and let $B\left(x_{i}, x_{j}\right)$ be one of the unit discs that contain $x_{i}$ and $x_{j}$ on its boundary. The shorter arc of $\partial B\left(x_{i}, x_{j}\right)$ forms an edge of $K_{n}$ if the entire set $X_{n}$ is contained in $B\left(x_{i}, x_{j}\right)$. Note that it may happen that the pair $x_{i}, x_{j}$ determines two edges of $K_{n}$ if the above condition holds for both unit discs that contain $x_{i}$ and $x_{j}$ on its boundary.

We recall that the Hausdorff distance $d_{\mathrm{H}}(A, B)$ of two nonempty compact sets $A, B \subset \mathbb{R}^{2}$ is

$$
d_{\mathrm{H}}(A, B):=\max \left\{\max _{a \in A} \min _{b \in B} d(a, b), \max _{b \in B} \min _{a \in A} d(a, b)\right\},
$$

where $d(a, b)$ is the Euclidean distance of $a$ and $b$.

First, we note that for the proof of Theorem 3, similar to [23], we may assume that the Hausdorff distance $d_{\mathrm{H}}\left(K, K_{n}\right)$ of $K$ and $K_{n}$ is at most $\varepsilon_{K}$, where $\varepsilon_{K}>0$ is a suitably chosen constant. This can be seen in the following way. Assume that $d_{\mathrm{H}}\left(K, K_{n}\right) \geq \varepsilon_{K}$. Then there exists a point $x$ on the boundary of $K_{n}$ such that $\varepsilon_{K} B^{2}+x \subset K$. There exists a supporting circle of $K_{n}$ through $x$ that determines a disc-cap of height at least $\varepsilon_{K}$. By the above remark, the probability content of this disc-cap is at least $c_{K}>0$, where $c_{K}$ is a suitable constant depending on $K$ and $\varepsilon_{K}$. Then

$$
\mathbb{P}\left(d_{\mathrm{H}}\left(K, K_{n}\right) \geq \varepsilon_{K}\right) \leq\left(1-c_{K}\right)^{n} .
$$

Our main tool in the variance estimates is the Efron-Stein inequality [14], which has previously been used to provide upper estimates on the variance of various geometric quantities associated with random polytopes in convex bodies; see [23], and for further references in this topic we recommend the recent surveys [1] and [30].

\section{Proof of Theorem 3}

We present the proof of the asymptotic upper bound on the variance of the vertex number in detail. Since the argument for the variance of the missed area is very similar, we only indicate the key steps in the last few paragraphs of this section. Our argument is similar to the one in [23, Sections 4 and 6]. The basic idea of the argument rests on the Efron-Stein inequality, which bounds the variance of a random variable (in our case the vertex number or the missed area) in terms of expectations. To calculate the involved expectations, we use some basic geometric properties of disc-caps and the integral transformation [18, pp. 907-909], see also [27]. Finally, the asymptotic estimate (11) in [13, p. 2290] for the order of magnitude of beta integrals yields the desired asymptotic upper bound.

For the number of vertices of $K_{n}$, the Efron-Stein inequality [14] states the following:

$$
\operatorname{var} f_{0}\left(K_{n}\right) \leq(n+1) \mathbb{E}\left(f_{0}\left(K_{n+1}\right)-f_{0}\left(K_{n}\right)\right)^{2} .
$$

Let $x$ be an arbitrary point of $K$ and let $x_{i} x_{j}$ be an edge of $K_{n}$. Following [23], we say that the edge $x_{i} x_{j}$ is visible from $x$ if $x$ is not contained in $K_{n}$ and it is not contained in the unit disc of the edge $x_{i} x_{j}$. For a point $x \in K \backslash K_{n}$, let $\mathcal{F}_{n}(x)$ denote the set of edges of $K_{n}$ that can be seen from $x$, and for $x \in K_{n}$, set $\mathcal{F}_{n}(x)=\varnothing$. Let $F_{n}(x)=\left|\mathcal{F}_{n}(x)\right|$. 
Let $x_{n+1}$ be a uniform random point in $K$ chosen independently from $X_{n}$. If $x_{n+1} \in K_{n}$ then $f_{0}\left(K_{n+1}\right)=f_{0}\left(K_{n}\right)$. If, on the other hand, $x_{n+1} \notin K_{n}$ then

$$
f_{0}\left(K_{n+1}\right)=f_{0}\left(K_{n}\right)+1-\left(F_{n}\left(x_{n+1}\right)-1\right)=f_{0}\left(K_{n}\right)-F_{n}\left(x_{n+1}\right)+2 .
$$

Therefore,

$$
\left|f_{0}\left(K_{n+1}\right)-f_{0}\left(K_{n}\right)\right| \leq 2 F_{n}\left(x_{n+1}\right),
$$

and, by the Efron-Stein jackknife inequality,

$$
\operatorname{var}\left(f_{0}\left(K_{n}\right)\right) \leq(n+1) \mathbb{E}\left(f_{0}\left(K_{n+1}\right)-f_{0}\left(K_{n}\right)\right)^{2} \leq 4(n+1) \mathbb{E}\left(F_{n}^{2}\left(x_{n+1}\right)\right) .
$$

Similar to [23], we introduce the following notation; see [23, p. 2147]. Let $I=\left(i_{1}, i_{2}\right), i_{1} \neq$ $i_{2}, i_{1}, i_{2} \in\{1,2, \ldots\}$ be an ordered pair of indices. Denote by $F_{I}$ the shorter arc of the unique unit circle incident with $x_{i_{1}}$ and $x_{i_{2}}$ on which $x_{i_{1}}$ follows $x_{i_{2}}$ in the positive cyclic ordering of the circle. Let $\mathbf{1}(A)$ denote the indicator function of the event $A$. For the sake of brevity, we use the notation $x_{1}, x_{2}, \ldots$ for the integration variables as well.

We wish to estimate the expectation $\mathbb{E}\left(F_{n}^{2}\left(x_{n+1}\right)\right)$ under the condition that $d_{\mathrm{H}}\left(K, K_{n}\right)<\varepsilon_{K}$. To compensate for the cases in which $d_{\mathrm{H}}\left(K, K_{n}\right) \geq \varepsilon_{k}$, using (8), we add an error term $O\left(\left(1-c_{K}\right)^{n}\right)$. Thus,

$$
\begin{aligned}
& \mathbb{E}\left(F_{n}\left(x_{n+1}\right)^{2}\right) \\
& =\frac{1}{A(K)^{n+1}} \int_{K} \int_{K^{n}}\left(\sum_{I} \mathbf{1}\left(F_{I} \in \mathcal{F}_{n}\left(x_{n+1}\right)\right)\right)^{2} \mathrm{~d} X_{n} \mathrm{~d} x_{n+1} \\
& =\frac{1}{A(K)^{n+1}} \int_{K} \int_{K^{n}}\left(\sum_{I} \mathbf{1}\left(F_{I} \in \mathcal{F}_{n}\left(x_{n+1}\right)\right)\right)\left(\sum_{J} \mathbf{1}\left(F_{J} \in \mathcal{F}_{n}\left(x_{n+1}\right)\right)\right) \mathrm{d} X_{n} \mathrm{~d} x_{n+1} \\
& \leq \frac{1}{A(K)^{n+1}} \sum_{I} \sum_{J} \int_{K} \int_{K^{n}} \mathbf{1}\left(F_{I} \in \mathcal{F}_{n}\left(x_{n+1}\right)\right) \mathbf{1}\left(F_{J} \in \mathcal{F}_{n}\left(x_{n+1}\right)\right) \\
& \quad \times \mathbf{1}\left(d_{\mathrm{H}}\left(K, K_{n}\right) \leq \varepsilon_{K}\right) \mathrm{d} X_{n} \mathrm{~d} x_{n+1}+O\left(\left(1-c_{K}\right)^{n}\right) .
\end{aligned}
$$

Choose $\varepsilon_{K}$ so small that $A\left(K \backslash K_{n}\right)<\delta$. Note that with this choice of $\varepsilon_{K}$ only one of the two shorter arcs determined by $x_{i_{1}}$ and $x_{i_{2}}$ can determine an edge of $K_{n}$.

Now we fix the number $k$ of common elements of $I$ and $J$, that is, $|I \cap J|=k$. Let $F_{1}$ denote one of the shorter arcs spanned by $x_{1}$ and $x_{2}$, and let $F_{2}$ be one of the shorter arcs determined by $x_{3-k}$ and $x_{4-k}$. Since the random points are independent, we have

$$
\begin{aligned}
(10) \ll & \frac{1}{A(K)^{n+1}} \sum_{k=0}^{2}\left(\begin{array}{l}
n \\
2
\end{array}\right)\left(\begin{array}{l}
2 \\
k
\end{array}\right)\left(\begin{array}{l}
n-2 \\
2-k
\end{array}\right) \\
& \times \int_{K} \int_{K^{n}} \mathbf{1}\left(F_{1} \in \mathcal{F}_{n}\left(x_{n+1}\right)\right) \mathbf{1}\left(F_{2} \in \mathcal{F}_{n}\left(x_{n+1}\right)\right) \\
& \times \mathbf{1}\left(d_{\mathrm{H}}\left(K, K_{n}\right) \leq \varepsilon_{K}\right) \mathrm{d} X_{n} \mathrm{~d} x_{n+1}+O\left(\left(1-c_{K}\right)^{n}\right) \\
\ll & \frac{1}{A(K)^{n+1}} \sum_{k=0}^{2} n^{4-k} \int_{K} \ldots \int_{K} \mathbf{1}\left(F_{1} \in \mathcal{F}_{n}\left(x_{n+1}\right)\right) \mathbf{1}\left(F_{2} \in \mathcal{F}_{n}\left(x_{n+1}\right)\right) \\
\quad & \times \mathbf{1}\left(d_{\mathrm{H}}\left(K, K_{n}\right) \leq \varepsilon_{K}\right) \mathrm{d} X_{n} \mathrm{~d} x_{n+1}+O\left(\left(1-c_{K}\right)^{n}\right) .
\end{aligned}
$$


Since the roles of $F_{1}$ and $F_{2}$ are symmetric, we may assume that diam $C_{+}\left(D_{1}\right) \geq$ diam $C_{+}\left(D_{2}\right)$, where $D_{1}=D_{-}\left(x_{1}, x_{2}\right)$ and $D_{2}=D_{-}\left(x_{3-k}, x_{4-k}\right)$ are the corresponding disc-caps, and $\operatorname{diam}(\cdot)$ denotes the diameter of a set. Thus,

$$
\begin{array}{rl}
(11) \ll \frac{1}{A(K)^{n+1}} \sum_{k=0}^{2} n^{4-k} \int_{K} \int_{K^{n}} & \mathbf{1}\left(F_{1} \in \mathcal{F}_{n}\left(x_{n+1}\right)\right) \\
& \times \mathbf{1}\left(F_{2} \in \mathcal{F}_{n}\left(x_{n+1}\right)\right) \mathbf{1}\left(\operatorname{diam} C_{+}\left(D_{1}\right) \geq \operatorname{diam} C_{+}\left(D_{2}\right)\right) \\
& \times \mathbf{1}\left(d_{\mathrm{H}}\left(K, K_{n}\right) \leq \varepsilon_{K}\right) \mathrm{d} X_{n} \mathrm{~d} x_{n+1}+O\left(\left(1-c_{K}\right)^{n}\right) .
\end{array}
$$

Clearly, $x_{n+1}$ is a common point of the disc-caps $D_{1}$ and $D_{2}$, so we may write

$$
\begin{array}{rl}
(12) \leq \frac{1}{A(K)^{n+1}} \sum_{k=0}^{2} n^{4-k} \int_{K} \int_{K^{n}} & \mathbf{1}\left(F_{1} \in \mathcal{F}_{n}\left(x_{n+1}\right)\right) \\
\times & \mathbf{1}\left(D_{1} \cap D_{2} \neq \varnothing\right) \mathbf{1}\left(\operatorname{diam} C_{+}\left(D_{1}\right) \geq \operatorname{diam} C_{+}\left(D_{2}\right)\right) \\
\times & \mathbf{1}\left(d_{\mathrm{H}}\left(K, K_{n}\right) \leq \varepsilon_{K}\right) \mathrm{d} X_{n} \mathrm{~d} x_{n+1}+O\left(\left(1-c_{K}\right)^{n}\right) .
\end{array}
$$

In order for $F_{1}$ to be an edge of $K_{n}$, it is necessary that $x_{5-k}, \ldots x_{n} \in K \backslash D_{1}$, and for $F_{1} \in \mathcal{F}_{n}\left(x_{n+1}\right) x_{n+1}$ must be in $D_{1}$. Therefore,

$(13) \ll \frac{1}{A(K)^{n+1}} \sum_{k=0}^{2} n^{4-k} \int_{K} \cdots \int_{K}\left(A(K)-A\left(D_{1}\right)\right)^{n-4+k} A\left(D_{1}\right)$

$$
\begin{aligned}
& \times \mathbf{1}\left(D_{1} \cap D_{2} \neq \varnothing\right) \mathbf{1}\left(\operatorname{diam} C_{+}\left(D_{1}\right) \geq \operatorname{diam} C_{+}\left(D_{2}\right)\right) \\
& \times \mathbf{1}\left(d_{\mathrm{H}}\left(K, K_{n}\right) \leq \varepsilon_{K}\right) \mathrm{d} x_{1} \cdots \mathrm{d} x_{4-k}+O\left(\left(1-c_{K}\right)^{n}\right) \\
& \ll \sum_{k=0}^{2} n^{4-k} \int_{K} \cdots \int_{K}\left(1-\frac{A\left(D_{1}\right)}{A(K)}\right)^{n-4+k} \frac{A\left(D_{1}\right)}{A(K)} \\
& \times \mathbf{1}\left(D_{1} \cap D_{2} \neq \varnothing\right) \mathbf{1}\left(\operatorname{diam} C_{+}\left(D_{1}\right) \geq \operatorname{diam} C_{+}\left(D_{2}\right)\right) \\
& \times \mathbf{1}\left(d_{\mathrm{H}}\left(K, K_{n}\right) \leq \varepsilon_{K}\right) \mathrm{d} x_{1} \cdots \mathrm{d} x_{4-k}+O\left(\left(1-c_{K}\right)^{n}\right) .
\end{aligned}
$$

Reitzner (see [23, pp. 2149-2150]) proved that if $D_{1} \cap D_{2} \neq \varnothing, d_{\mathrm{H}}\left(K, K_{n}\right) \leq \varepsilon_{K}$, and diam $C_{+}\left(D_{1}\right) \geq \operatorname{diam} C_{+}\left(D_{2}\right)$ then there exists a constant $\bar{c}$ (depending only on $K$ ) such that $C_{+}\left(D_{2}\right) \subset \bar{c}\left(C_{+}\left(D_{1}\right)-x_{D_{1}}\right)+x_{D_{1}}$, where $x_{D_{1}}$ is the vertex of $D_{1}$. Combining this with Claim 1 we find that there is a constant $c_{1}$ depending only on $K$, such that $D_{2} \subset c_{1}\left(D_{1}-x_{D_{1}}\right)+x_{D_{1}}$. Hence, $A\left(D_{2}\right) \leq c_{1}^{2} A\left(D_{1}\right)$ and, therefore,

$$
\begin{aligned}
\int_{K} \ldots & \int_{K} \mathbf{1}\left(D_{1} \cap D_{2} \neq \varnothing\right) \mathbf{1}\left(\operatorname{diam} C_{+}\left(D_{1}\right) \geq \operatorname{diam} C_{+}\left(D_{2}\right)\right) \\
& \times \mathbf{1}\left(d_{\mathrm{H}}\left(K, K_{n}\right) \leq \varepsilon_{K}\right) \mathrm{d} x_{3} \cdots \mathrm{d} x_{4-k} \\
\ll & A\left(D_{1}\right)^{2-k} .
\end{aligned}
$$


We continue by estimating (14) term by term (omitting the $O\left(\left(1-c_{K}\right)^{n}\right)$ term).

$$
\begin{aligned}
n^{4-k} \int_{K} \cdots \int_{K} & \left(1-\frac{A\left(D_{1}\right)}{A(K)}\right)^{n-4+k} \frac{A\left(D_{1}\right)}{A(K)} \mathbf{1}\left(D_{1} \cap D_{2} \neq \varnothing\right) \\
& \times \mathbf{1}\left(\operatorname{diam} C_{+}\left(D_{1}\right) \geq \operatorname{diam} C_{+}\left(D_{2}\right)\right) \mathbf{1}\left(d_{\mathrm{H}}\left(K, K_{n}\right) \leq \varepsilon_{K}\right) \mathrm{d} x_{1} \cdots \mathrm{d} x_{4-k} \\
\ll & n^{4-k} \int_{K} \int_{K}\left(1-\frac{A\left(D_{1}\right)}{A(K)}\right)^{n-4+k}\left(\frac{A\left(D_{1}\right)}{A(K)}\right)^{3-k} \mathbf{1}\left(d_{\mathrm{H}}\left(K, K_{n}\right) \leq \varepsilon_{K}\right) \mathrm{d} x_{1} \mathrm{~d} x_{2} .
\end{aligned}
$$

Now, we use the following parametrization of $\left(x_{1}, x_{2}\right)$ the same way as in [18] to transform the integral. Let

$$
\left(x_{1}, x_{2}\right)=\Phi\left(u, t, u_{1}, u_{2}\right)
$$

where $u, u_{1}, u_{2} \in S^{1}$, and $0 \leq t \leq t_{0}(u)$ are chosen such that

$$
D(u, t)=D_{1}=D_{-}\left(x_{1}, x_{2}\right) \quad \text { and } \quad\left(x_{1}, x_{2}\right)=\left(x_{u}-(1+t) u+u_{1}, x_{u}-(1+t) u+u_{2}\right) .
$$

More information on this transformation can be found in [18, pp. 907-909]. Here we just recall that the Jacobian of $\Phi$ is

$$
|J \Phi|=\left(1+t-\frac{1}{\kappa\left(x_{u}\right)}\right)\left|u_{1} \times u_{2}\right|,
$$

where $u_{1} \times u_{2}$ denotes the cross product of $u_{1}$ and $u_{2}$.

Let $L(u, t)=\partial D_{1} \cap$ int $K$ then we obtain

$$
\begin{aligned}
&(15) \ll n^{4-k} \int_{S^{1}} \int_{0}^{t^{*}(u)} \int_{L(u, t)} \int_{L(u, t)}\left(1-\frac{A(u, t)}{A(K)}\right)^{n-4+k}\left(\frac{A(u, t)}{A(K)}\right)^{3-k} \times\left(1+t-\frac{1}{\kappa\left(x_{u}\right)}\right)\left|u_{1} \times u_{2}\right| \mathrm{d} u_{1} \mathrm{~d} u_{2} \mathrm{~d} t \mathrm{~d} u \\
&=n^{4-k} \int_{S^{1}} \int_{0}^{t^{*}(u)}\left(1-\frac{A(u, t)}{A(K)}\right)^{n-4+k}\left(\frac{A(u, t)}{A(K)}\right)^{3-k} \\
& \times\left(1+t-\frac{1}{\kappa\left(x_{u}\right)}\right)(\ell(u, t)-\sin \ell(u, t)) \mathrm{d} t \mathrm{~d} u .
\end{aligned}
$$

From now on the evaluation follows in a standard way. First, we split the domain of integration with respect to $t$ into two parts. Let $h(n)=(c \ln n / n)^{2 / 3}$, where $c>0$ is a sufficiently large absolute constant. Using (7), it follows that $A(u, t) \geq \gamma t^{3 / 2}$ uniformly in $u \in S^{1}$; hence,

$$
\begin{aligned}
n^{4-k} \int_{S^{1}} \int_{h(n)}^{t^{*}(u)}\left(1-\frac{A(u, t)}{A(K)}\right)^{n-4+k}\left(\frac{A(u, t)}{A(K)}\right)^{3-k} \\
\quad \times\left(1+t-\frac{1}{\kappa\left(x_{u}\right)}\right)(\ell(u, t)-\sin \ell(u, t)) \mathrm{d} t \mathrm{~d} u \\
\ll n^{4-k} \int_{S^{1}} \int_{h(n)}^{t^{*}(u)}\left(1-\frac{A(u, t)}{A(K)}\right)^{n-4+k} \mathrm{~d} t \mathrm{~d} u \\
\ll n^{4-k} \int_{S^{1}} \int_{h(n)}^{t^{*}(u)}\left(1-\frac{\gamma t^{3 / 2}}{A(K)}\right)^{n-4+k} \mathrm{~d} t \mathrm{~d} u \\
\ll n^{4-k}\left(1-\frac{\gamma h(n)^{3 / 2}}{A(K)}\right)^{n-4+k}
\end{aligned}
$$




$$
\begin{aligned}
& =n^{4-k}\left(1-\frac{\gamma(c \ln n)}{n A(K)}\right)^{n-4+k} \\
& \ll n^{-2 / 3}
\end{aligned}
$$

if $\gamma c / A(K)$ is sufficiently large.

Therefore, it is enough to estimate the following part of (16):

$$
\begin{aligned}
n^{4-k} \int_{S^{1}} \int_{0}^{h(n)} & \left(1-\frac{A(u, t)}{A(K)}\right)^{n-4+k}\left(\frac{A(u, t)}{A(K)}\right)^{3-k} \\
& \times\left(1+t-\frac{1}{\kappa\left(x_{u}\right)}\right)(\ell(u, t)-\sin \ell(u, t)) \mathrm{d} t \mathrm{~d} u .
\end{aligned}
$$

Using (7) and the Taylor series of the sine function, we obtain $\ell(u, t)-\sin \ell(u, t) \ll t^{3 / 2}$. Since $\kappa(x)>1$ for all $x \in \partial K$, it follows that $0<1+t-\kappa\left(x_{u}\right)^{-1} \ll 1$. We also use (7) to estimate $A(u, t)$, similarly as before. Assuming that $n$ is large enough, we obtain

$$
\begin{aligned}
(17) & \ll n^{4-k} \int_{S^{1}} \int_{0}^{h(n)}\left(1-\frac{\gamma t^{3 / 2}}{A(K)}\right)^{n-4+k}\left(t^{3 / 2}\right)^{3-k} \cdot 1 \cdot t^{3 / 2} \mathrm{~d} t \mathrm{~d} u \\
& \ll n^{4-k} \int_{0}^{h(n)}\left(1-\frac{\gamma t^{3 / 2}}{A(K)}\right)^{n-4+k} t^{(12-3 k) / 2} \mathrm{~d} t \\
& \ll n^{-2 / 3},
\end{aligned}
$$

where the last inequality follows directly from [13, Equation (11), p. 2290]. Together with (9), this yields the desired upper estimate for var $f_{0}\left(K_{n}\right)$.

As the argument for the case of the missing area is very similar, we only highlight the major steps.

Again, we use the Efron-Stein inequality [14], which states the following for the missed area:

$$
\operatorname{var} A\left(K \backslash K_{n}\right) \leq(n+1) \mathbb{E}\left(A\left(K_{n+1}\right)-A\left(K_{n}\right)\right)^{2} .
$$

Therefore, we need to estimate $\mathbb{E}\left(A\left(K_{n+1}\right)-A\left(K_{n}\right)\right)^{2}$. Following the ideas of Reitzner [23], we see that

$$
\begin{gathered}
\mathbb{E}\left(A\left(K_{n+1}\right)-A\left(K_{n}\right)\right)^{2} \ll \sum_{I} \sum_{J} \int_{K} \int_{K^{n}} \mathbf{1}\left(F_{1} \in \mathcal{F}_{n}\left(x_{n+1}\right)\right) A\left(D_{1}\right) \mathbf{1}\left(F_{2} \in \mathcal{F}_{n}\left(x_{n+1}\right)\right) A\left(D_{2}\right) \\
\times \mathbf{1}\left(d_{\mathrm{H}}\left(K, K_{n}\right) \leq \varepsilon_{K}\right) \mathrm{d} X_{n} \mathrm{~d} x_{n+1}
\end{gathered}
$$

From here, we closely follow the proof of (2), the only major difference being the extra $A\left(D_{1}\right) A\left(D_{2}\right) \leq A^{2}\left(D_{1}\right)$ factor in the integrand. After similar calculations as for the vertex number, we obtain

$$
\begin{aligned}
&(18) \ll n^{4-k} \int_{S^{1}} \int_{0}^{h(n)}\left(1-\frac{A(u, t)}{A(K)}\right)^{n-4+k}\left(\frac{A(u, t)}{A(K)}\right)^{5-k} \\
& \quad \times\left(1+t-\frac{1}{\kappa\left(x_{u}\right)}\right)(\ell(u, t)-\sin \ell(u, t)) \mathrm{d} t \mathrm{~d} u . \\
& \ll n^{4-k} \int_{0}^{h(n)}\left(1-c_{K} t^{3 / 2}\right)^{n-4+k} t^{(20-3 k) / 2} \mathrm{~d} t \\
& \ll n^{-8 / 3},
\end{aligned}
$$

which proves (3) (the missing factor $n$ comes from the Efron-Stein inequality). 


\section{The case of the circle}

In this section we prove Theorem 4. In particular, we give a detailed proof of the estimate (4) for the variance of the number of vertices of the random disc-polygon. The case of the missed area (5) is very similar.

Without loss of generality, we may assume that $K=B^{2}$, and that $r=1$.

We begin by recalling from [18] that for any $u \in S^{1}$ and $0 \leq t \leq 2$, it holds that

$$
\ell(u, t)=2 \arcsin \sqrt{1-\frac{1}{4} t^{2}}, \quad \text { and } \quad A(u, t)=A(t)=t \sqrt{1-\frac{1}{4} t^{2}}+2 \arcsin \frac{1}{2} t .
$$

Proof of Theorem 4 (Equation (4)). From (1) and Chebyshev's inequality, it follows that

$$
1=\mathbb{P}\left(\left|f_{0}\left(K_{n}^{1}\right)-\frac{\pi^{2}}{2}\right|>0.05\right) \leq \frac{\operatorname{var}\left(f_{0}\left(K_{n}^{1}\right)\right)}{0.05^{2}} ;
$$

thus,

$$
\operatorname{var}\left(f_{0}\left(K_{n}^{1}\right)\right) \geq 0.05^{2} .
$$

This proves that $\operatorname{var}\left(f_{0}\left(K_{n}^{1}\right)\right) \gg$ constant.

In order to prove the asymptotic upper bound in (4), we use a modified version of the argument of the previous section. With the same notation as in Section 3, the Efron-Stein inequality for the vertex number yields that

$$
\operatorname{var}\left(f_{0}\left(K_{n}^{1}\right)\right) \ll n \mathbb{E}\left(F_{n}\left(x_{n+1}\right)\right)^{2} .
$$

Following a similar line of argument as above, we obtain

$$
\begin{aligned}
& n \mathbb{E}\left(F_{n}\left(x_{n+1}\right)\right)^{2} \\
& =\frac{n}{\pi^{n+1}} \int_{\left(B^{2}\right)^{n+1}}\left(\sum_{I} \mathbf{1}\left(F_{I} \in \mathcal{F}_{n}\left(x_{n+1}\right)\right)\right) \\
& \quad \times\left(\sum_{J} \mathbf{1}\left(F_{J} \in \mathcal{F}_{n}\left(x_{n+1}\right)\right)\right) \mathrm{d} x_{1} \cdots \mathrm{d} x_{n} \mathrm{~d} x_{n+1} \\
& \leq \frac{n}{\pi^{n+1}} \sum_{I} \sum_{J} \int_{\left(B^{2}\right)^{n+1}} \mathbf{1}\left(F_{I} \in \mathcal{F}_{n}\left(x_{n+1}\right)\right) \mathbf{1}\left(F_{J} \in \mathcal{F}_{n}\left(x_{n+1}\right)\right) \mathrm{d} x_{1} \cdots \mathrm{d} x_{n} \mathrm{~d} x_{n+1} .
\end{aligned}
$$

Now, let $|I \cap J|=k$, where $k=0,1,2$, and let $F_{1}=x_{1} x_{2}$ and $F_{2}=x_{3-k} x_{4-k}$. By the independence of the random points (and by also taking into account their order), we have

$$
\begin{aligned}
(19) & \ll \frac{n}{\pi^{n+1}} \sum_{k=0}^{2}\left(\begin{array}{l}
n \\
2
\end{array}\right)\left(\begin{array}{l}
2 \\
k
\end{array}\right)\left(\begin{array}{l}
n-2 \\
2-k
\end{array}\right) \int_{\left(B^{2}\right)^{n+1}} \mathbf{1}\left(F_{1} \in \mathcal{F}_{n}\left(x_{n+1}\right)\right) \\
& \times \mathbf{1}\left(F_{2} \in \mathcal{F}_{n}\left(x_{n+1}\right)\right) \mathrm{d} x_{1} \cdots \mathrm{d} x_{n} \mathrm{~d} x_{n+1} . \\
& \ll \frac{1}{\pi^{n+1}} \sum_{k=0}^{2} n^{5-k} \int_{\left(B^{2}\right)^{n+1}} \mathbf{1}\left(F_{1} \in \mathcal{F}_{n}\left(x_{n+1}\right)\right) \mathbf{1}\left(F_{2} \in \mathcal{F}_{n}\left(x_{n+1}\right)\right) \mathrm{d} x_{1} \cdots \mathrm{d} x_{n} \mathrm{~d} x_{n+1} .
\end{aligned}
$$


By symmetry, we may also assume that $A\left(D_{1}\right) \geq A\left(D_{2}\right)$; therefore,

$$
\begin{array}{rl}
(20) \ll \sum_{k=0}^{2} n^{5-k} \int_{\left(B^{2}\right)^{n+1}} & \mathbf{1}\left(F_{1} \in \mathcal{F}_{n}\left(x_{n+1}\right)\right) \mathbf{1}\left(F_{2} \in \mathcal{F}_{n}\left(x_{n+1}\right)\right) \\
\times \mathbf{1}\left(A\left(D_{1}\right) \geq A\left(D_{2}\right)\right) \mathrm{d} x_{1} \cdots \mathrm{d} x_{n} \mathrm{~d} x_{n+1} .
\end{array}
$$

By integrating with respect to $x_{5-k}, \ldots, x_{n}$ and $x_{n+1}$, we obtain

$(21) \ll \sum_{k=0}^{2} n^{5-k} \int_{B^{2}} \cdots \int_{B^{2}}\left(1-\frac{A\left(D_{1}\right)}{\pi}\right)^{n-4+k} \frac{A\left(D_{1}\right)}{\pi} \mathbf{1}\left(A\left(D_{1}\right) \geq A\left(D_{2}\right)\right) \mathrm{d} x_{1} \cdots \mathrm{d} x_{4-k}$

If $A\left(D_{1}\right) \geq A\left(D_{2}\right)$ then $D_{2}$ is fully contained in the circular annulus whose width is equal to the height of the disc-cap $D_{1}$. The area of this annulus is not more than $4 A\left(D_{1}\right)$. Therefore,

$$
(22) \ll \sum_{k=0}^{2} n^{5-k} \int_{B^{2}} \int_{B^{2}}\left(1-\frac{A\left(D_{1}\right)}{\pi}\right)^{n-4+k} A\left(D_{1}\right)^{3-k} \mathrm{~d} x_{1} \mathrm{~d} x_{2} .
$$

As is common in these arguments, we may assume that $A\left(D_{1}\right) / \pi<c \log n / n$ for some suitable constant $c>0$ that will be determined later. To see this, let $A\left(D_{1}\right) / \pi \geq c \log n / n$. Then

$$
\begin{aligned}
\left(1-\frac{A\left(D_{1}\right)}{\pi}\right)^{n-4+k} A\left(D_{1}\right)^{3-k} & \leq\left(\frac{\pi c \log n}{n}\right)^{3-k} \exp \left(-\frac{c(n-4+k) \log n}{n}\right) \\
& \ll\left(\frac{\log n}{n}\right)^{3-k} n^{-c} \\
& \ll n^{-c} .
\end{aligned}
$$

If $c>0$ is sufficiently large then the contribution of the $A\left(D_{1}\right) / \pi \geq c \log n / n$ case is $O\left(n^{-1}\right)$. Thus,

$$
\begin{aligned}
n \mathbb{E}\left(F_{n}\left(x_{n+1}\right)\right) \ll \sum_{k=0}^{2} n^{5-k} \int_{B^{2}} \int_{B^{2}} & \left(1-\frac{A\left(D_{1}\right)}{\pi}\right)^{n-4+k} A\left(D_{1}\right)^{3-k} \\
& \times \mathbf{1}\left(A\left(D_{1}\right) \leq \frac{c \log n}{n}\right) \mathrm{d} x_{1} \mathrm{~d} x_{2}+O\left(n^{-1}\right) .
\end{aligned}
$$

Now, we use the same type of reparametrization as in the previous section. Let $\left(x_{1}, x_{2}\right)=$ $\left(-t u_{1},-t u_{2}\right), u \in S^{1}$, and $0 \leq t<c^{*} \log n / n$. Then

$$
\begin{gathered}
(23) \ll \sum_{k=0}^{2} n^{5-k} \int_{S^{1}} \int_{0}^{c^{*} \log n / n} \int_{S^{1}} \int_{S^{1}}\left(1-\frac{A(u, t)}{\pi}\right)^{n-4+k} A(u, t)^{3-k} \\
\times t\left|u_{1} \times u_{2}\right| \mathrm{d} u_{1} \mathrm{~d} u_{2} \mathrm{~d} u \mathrm{~d} t+O\left(n^{-1}\right) \\
\ll \sum_{k=0}^{2} n^{5-k} \int_{0}^{c^{*} \log n / n}\left(1-\frac{A(u, t)}{\pi}\right)^{n-4+k} A(u, t)^{3-k} \\
\times t(l(t)-\sin l(t)) \mathrm{d} t+O\left(n^{-1}\right) .
\end{gathered}
$$


Using the fact that $l(t) \rightarrow \pi$ as $t \rightarrow 0^{+}$, and the Taylor series of $V(u, t)$ at $t=0$, we find that there exists a constant $\omega>0$ such that

$$
(24) \ll \sum_{k=0}^{2} n^{5-k} \int_{0}^{c^{*} \log n / n}(1-\omega t)^{n-4+k} t^{4-k} \mathrm{~d} t+O\left(n^{-1}\right) .
$$

Now, using a formula for the asymptotic order of beta integrals (see [13, Equation (11), p. 2290]), we obtain

$$
(25) \ll \sum_{k=0}^{2} n^{5-k} n^{-(5-k)}+O\left(n^{-1}\right) \ll \text { constant, }
$$

which completes the proof of the upper bound in (4).

In order to prove the asymptotic upper bound (5), only slight modifications are needed in the above argument.

\section{A circumscribed model}

In this section we consider circumscribed random disc-polygons. Let $K \subset \mathbb{R}^{2}$ be a convex disc with $C_{+}^{2}$ smooth boundary, and $r \geq \kappa_{\mathrm{m}}^{-1}$. Consider the following set:

$$
K^{*, r}=\left\{x \in \mathbb{R}^{2} \mid K \subset r B^{2}+x\right\},
$$

which is also called the $r$-hyperconvex dual, or $r$-dual for short, of $K$. It is known that $K^{*, r}$ is a convex disc with $C_{+}^{2}$ boundary, and it also has the property that the curvature is at least $1 / r$ at every boundary point. See [19] and the references therein for further details.

For $u \in S^{1}$, let $x(K, u) \in \partial K\left(x\left(K^{*, r}, u\right) \in \partial K^{*, r}\right.$, respectively) be the unique point on $\partial K\left(\partial K^{*, r}\right.$, respectively), where the outer unit normal to $K$ (respectively, $\left.K^{*, r}\right)$ is $u$. For a convex disc $K \subset \mathbb{R}^{2}$ with $o \in$ int $K$, let $h_{K}(u)=\max _{x \in K}\langle x, u\rangle$ denote the support function of $K$. Let $\operatorname{per}(\cdot)$ denote the perimeter.

In the following lemma we collect some results from [19, Section 2].

Lemma 2. (Fodor et al. [19].) With the notation above

(i) $h_{K}(u)+h_{K^{*}, r}(-u)=r$ for any $u \in S^{1}$;

(ii) $\kappa_{K}^{-1}(x(u, K))+\kappa_{K^{*, r}}^{-1}\left(x\left(-u, K^{*, r}\right)\right)=r$ for any $u \in S^{1}$;

(iii) $\operatorname{per}(K)+\operatorname{per}\left(K^{*, r}\right)=2 r \pi$;

(iv) $A\left(K^{*, r}\right)=A(K)-r \operatorname{per}(K)+r^{2} \pi$.

Now we turn to the probability model. Let $K$ be a convex disc with $C_{+}^{2}$ boundary, and let $r>\kappa_{\mathrm{m}}^{-1}$ as before. Let $X_{n}=\left\{x_{1}, \ldots, x_{n}\right\}$ be a sample of $n$ independent random points chosen from $K^{*, r}$ according to the uniform probability distribution, and define

$$
K_{(n)}^{*, r}=\bigcap_{x \in X_{n}} r B^{2}+x
$$

where $K_{(n)}^{*, r}$ is a random disc-polygon that contains $K$. Observe that, by definition $K_{(n)}^{*, r}=$ $\left(\operatorname{conv}_{r}\left(X_{n}\right)\right)^{*, r}$, and, consequently, $f_{0}\left(K_{(n)}^{*, r}\right)=f_{0}\left(\operatorname{conv}_{r}\left(X_{n}\right)\right)$. We note that this is a very natural approach to define a random disc-polygon that is circumscribed about $K$ that has no 
clear analogy in classical convexity. (If we take the limit as $r \rightarrow \infty$, the underlying probability measures do not converge.) The model is of special interest in the $K=K^{*, r}$ case, which happens exactly when $K$ is of constant width $r$.

Theorem 6. Assume that $K$ has $C_{+}^{2}$ boundary, and let $r>\kappa_{\mathrm{m}}^{-1}$. With the notation above

$$
\lim _{n \rightarrow \infty} \mathbb{E}\left(f_{0}\left(K_{(n)}^{*, r}\right)\right) n^{-1 / 3}=\sqrt[3]{\frac{2 r}{3\left(A(K)-r \operatorname{per}(K)+r^{2} \pi\right)}} \Gamma\left(\frac{5}{3}\right) \int_{\partial K}\left(\kappa(x)-\frac{1}{r}\right)^{2 / 3} \mathrm{~d} x .
$$

Furthermore, if $K$ has $C_{+}^{5}$ boundary then

$$
\begin{aligned}
\lim _{n \rightarrow \infty} n^{2 / 3}\left(\operatorname{per} K_{(n)}^{*, r}-\operatorname{per} K\right)= & \frac{\left(12\left(A(K)-r \operatorname{per}(K)+r^{2} \pi\right)\right)^{2 / 3}}{36} \Gamma\left(\frac{2}{3}\right) \\
& \times r^{-2 / 3} \int_{\partial K}\left(\kappa(x)-\frac{1}{r}\right)^{-1 / 3}\left(4 \kappa(x)-\frac{1}{r}\right) \mathrm{d} x, \\
\lim _{n \rightarrow \infty} n^{2 / 3} A\left(K_{(n)}^{*, r} \backslash K\right)= & \frac{\left(12\left(A(K)-r \operatorname{per}(K)+r^{2} \pi\right)\right)^{2 / 3}}{12} \\
& \times \Gamma\left(\frac{2}{3}\right) r^{-2 / 3} \int_{\partial K}\left(\kappa(x)-\frac{1}{r}\right)^{-1 / 3} \mathrm{~d} x .
\end{aligned}
$$

Proof. From Lemma 2, it follows that $K^{*, r}$ has also $C_{+}^{2}$ boundary. As $f_{0}\left(K_{(n)}^{*, r}\right)=$ $f_{0}\left(\operatorname{conv}_{r}\left(X_{n}\right)\right)$, from [18, Theorem 1.1], we immediately obtain

$$
\lim _{n \rightarrow \infty} \mathbb{E}\left(f_{0}\left(K_{(n)}^{*, r}\right)\right) n^{-1 / 3}=\sqrt[3]{\frac{2}{3 A\left(K^{*, r}\right)}} \Gamma\left(\frac{5}{3}\right) \int_{\partial K^{*, r}}\left(\kappa(x)-\frac{1}{r}\right)^{1 / 3} \mathrm{~d} x .
$$

Using Lemma 2, we proceed as follows:

$$
\begin{aligned}
\int_{\partial K^{*, r}} & \left(\kappa(x)-\frac{1}{r}\right)^{1 / 3} \mathrm{~d} x \\
= & \int_{S^{1}} \frac{\left(\kappa\left(x\left(K^{*, r}, u\right)\right)-1 / r\right)^{1 / 3}}{\kappa\left(x\left(K^{*, r}, u\right)\right)} \mathrm{d} u \\
= & \int_{S^{1}}\left[\left(\frac{\kappa(x(K,-u))}{r \kappa(x(K,-u))-1}-\frac{1}{r}\right)^{1 / 3}\right]\left[\frac{\kappa(x(K,-u))}{r \kappa(x(K,-u))-1}\right]^{-1} \mathrm{~d} u \\
= & \int_{S^{1}} r^{1 / 3} \frac{(\kappa(x(K, u))-1 / r)^{2 / 3}}{\kappa(x(K, u))} \mathrm{d} u \\
= & r^{1 / 3} \int_{\partial K}\left(\kappa(x)-\frac{1}{r}\right)^{2 / 3} \mathrm{~d} x
\end{aligned}
$$

Together with Lemma 2, this proves (26).

The rest of the theorem can be proved similarly, by using [18, Theorems 1.1 and 1.2], and Lemma 2.

As an obvious consequence of Theorem 3, Lemma 2, and the definition of $K_{(n)}^{*, r}$, we obtain the following corollary. 
Corollary 1. Assume that $K$ has $C_{+}^{2}$ boundary, and let $r>\kappa_{\mathrm{m}}^{-1}$. With the notation above

$$
\operatorname{var}\left(f_{0}\left(K_{(n)}^{*, r}\right)\right) \ll n^{1 / 3}
$$

Remark 1. We note that if $K$ is a convex disc of constant width $r$ then $K^{*, r}=K$ (see, for example, [19]), and similar calculations to those in the proof of Theorem 6 provide some interesting integral formulas. For example, for a real $p$, we obtain

$$
\int_{\partial K}\left(\kappa(x)-\frac{1}{r}\right)^{p} \mathrm{~d} x=r^{1-2 p} \int_{\partial K}\left(\kappa(x)-\frac{1}{r}\right)^{1-p} \mathrm{~d} x .
$$

\section{Acknowledgements}

The research of the authors was partially supported by the National Research, Development and Innovation Office of Hungary (grant number NKFIH 116451). V. Vígh was supported by the János Bolyai Research Scholarship of the Hungarian Academy of Sciences. This research was also supported by the EU-funded Hungarian grant EFOP-3.6.2-16-2017-00015.

\section{References}

[1] Bárány, I. (2008). Random points and lattice points in convex bodies. Bull. Amer. Math. Soc. 45, 339-365.

[2] BÁrÁNy, I. ANd Reitzner, M. (2010). On the variance of random polytopes. Adv. Math. 225, 1986-2001.

[3] Bárány, I. ANd Steiger, W. (2013). On the variance of random polygons. Comput. Geom. 46, $173-180$.

[4] Bárány, I. And Vu, V. H. (2007). Central limit theorems for Gaussian polytopes. Ann. Prob. 35, $1593-1621$.

[5] BÁrÁNY, I., Fodor, F. AND VÍGH, V. (2010). Intrinsic volumes of inscribed random polytopes in smooth convex bodies. Adv. Appl. Prob. 42, 605-619.

[6] Bárány, I., Hug, D., Reitzner, M. and Schneider, R. (2017). Random points in halfspheres. Random Structures Algorithms 46, 3-22.

[7] BezdeK, K. (2018). From $r$-dual sets to uniform contractions. Aequationes Math. 92, 123-134.

[8] Bezdek, K. And Connelly, R. (2002). Pushing disks apart-the Kneser-Poulsen conjecture in the plane. J. Reine Angew. Math. 553, 221-236.

[9] Bezdek, K. And Naszódi, M. (2018). The Kneser-Poulsen conjecture for special contractions. Discrete Comput. Geom. 60, 967-980.

[10] Bezdek, K., Lángi, Z., Naszódi, M. and Papez, P. (2007). Ball-polyhedra. Discrete Comput. Geom. 38, 201-230.

[11] BöröczKy, K. J. AND SCHneIder, R. (2010). The mean width of circumscribed random polytopes. Canad. Math. Bull. 53, 614-628.

[12] BöröczKy, K. J., Fodor, F. AND Hug, D. (2010). The mean width of random polytopes circumscribed around a convex body. J. Lond. Math. Soc. 81, 499-523.

[13] Böröczky, K. J., Fodor, F., Reitzner, M. And VíGH, V. (2009). Mean width of random polytopes in a reasonably smooth convex body. J. Multivariate Anal. 100, 2287-2295.

[14] Efron, B. And Stein, C. (1981). The jackknife estimate of variance. Ann. Statist. 9, 586-596.

[15] Fejes Tóth, G. ANd Fodor, F. (2015). Dowker-type theorems for hyperconvex discs. Period. Math. Hungar. 70, 131-144.

[16] Fodor, F. AND Vígh, V. (2012). Disc-polygonal approximations of planar spindle convex sets. Acta Sci. Math. 78, 331-350.

[17] Fodor, F., Hug, D. AND Ziebarth, I. (2016). The volume of random polytopes circumscribed around a convex body. Mathematika 62, 283-306.

[18] Fodor, F., Kevei, P. And Vígh, V. (2014). On random disc polygons in smooth convex discs. Adv. Appl. Prob. 46, 899-918.

[19] Fodor, F., Kurusa, Á. And VíGH, V. (2016). Inequalities for hyperconvex sets. Adv. Geom. 16, $337-348$.

[20] Jahn, T., Martini, H. And Richter, C. (2017). Ball convex bodies in Minkowski spaces. Pacific J. Math. 289, 287-316.

[21] MaYer, A. E. (1935). Eine Überkonvexität. Math. Z. 39, 511-531.

[22] Paouris, G. AND Pivovarov, P. (2017). Random ball-polyhedra and inequalities for intrinsic volumes. Monatsh. Math. 182, 709-729.

[23] ReitZner, M. (2003). Random polytopes and the Efron-Stein jackknife inequality. Ann. Prob. 31, $2136-2166$. 
[24] Reitzner, M. (2005). Central limit theorems for random polytopes. Prob. Theory Relat. Fields 133, $483-507$.

[25] RÉnYi, A. And Sulanke, R. (1963). Über die konvexe Hülle von $n$ zufällig gewählten Punkten. Z. Wahrscheinlichkeitsth. 2, 75-84.

[26] Rényi, A. and Sulanke, R. (1964). Über die konvexe Hülle von $n$ zufällig gewählten Punkten, II. Z. Wahrscheinlichkeitsth. 3, 138-147.

[27] Santaló, L. A. (1946). On plane hyperconvex figures. Summa Brasil. Math. 1, 221-239.

[28] Schneider, R. (2008). Recent results on random polytopes. Boll. Unione Mat. Ital. 1, 17-39.

[29] Schneider, R. (2014). Convex Bodies: The Brunn-Minkowski Theory, 2nd edn. Cambridge University Press.

[30] SchneIDer, R. (2017). Discrete aspects of stochastic geometry. In Handbook of Discrete and Computational Geometry, 3rd edn. CRC Press, Boca Raton, FL, pp. 299-329.

[31] SChneider, R. AND WeIL, W. (2008). Stochastic and Integral Geometry. Springer, Berlin.

[32] Schreiber, T. AND Yukich, J. E. (2008). Variance asymptotics and central limit theorems for generalized growth processes with applications to convex hulls and maximal points. Ann. Prob. 36, 363-396.

[33] Thäle, C., Turchi, N. and Wespi, F. (2018). Random polytopes: central limit theorems for intrinsic volumes. Proc. Amer. Math. Soc. 146, 3063-3071.

[34] Turchi, N. AND WeSPI, F. (2018). Limit theorems for random polytopes with vertices on convex surfaces. Adv. Appl. Prob. 50, 1227-1245.

[35] Vu, V. H. (2005). Sharp concentration of random polytopes. Geom. Funct. Anal. 15, 1284-1318.

[36] Vu, V. H. (2006). Central limit theorems for random polytopes in a smooth convex set. Adv. Math. 207, $221-243$.

[37] Weil, W. AND Wieacker, J. A. (1993). Stochastic geometry. In Handbook of Convex Geometria, Vol. B, NorthHolland, Amsterdam, pp. 1391-1438. 